\title{
Deuteron beam polarimeter at Nuclotron internal target
}

\author{
Ya.T. Skhomenko ${ }^{1,2, *}$, V.P. Ladygin ${ }^{1}$, Yu.V. Gurchin ${ }^{1}$, A.Yu. Isupov ${ }^{1}$, M. Janek ${ }^{3}$, \\ J.-T. Karachuk ${ }^{1,4}$, A.N. Khrenov ${ }^{1}$, P.K. Kurilkin ${ }^{1}$, A.N. Livanov ${ }^{1}$, S.M. Piyadin ${ }^{1}$, \\ S.G. Reznikov ${ }^{1}$, A.A. Terekhin ${ }^{1}$, A.V. Tishevsky ${ }^{1}$, A.V. Averyanov', A.S. Belov, \\ E.V. Chernykh ${ }^{1}$, D. Enache ${ }^{4}$, V.V. Fimushkin ${ }^{1}$, D.O. Krivenkov ${ }^{1}$, I.E. Vnukov ${ }^{2}$, and \\ I.S. Volkov ${ }^{1,2}$ \\ (DSS collaboration) \\ ${ }^{1}$ Joint Institute for Nuclear Research, Dubna, Russia \\ ${ }^{2}$ Belgorod State National Research University, Belgorod, Russia \\ ${ }^{3}$ Physics Department, University of Zilina, Zilina, Slovakia \\ ${ }^{4}$ National Institute for R\&D in Electrical Engineering ICPE-CA, Bucharest, Romania \\ ${ }^{5}$ Institute of Nuclear Physics, Moscow, Russia
}

\begin{abstract}
The current deuteron beam polarimetry at the Nuclotron is provided by the Internal Target polarimeter based on the use of the asymmetry in dpelastic scattering at large angles in the $\mathrm{cms}$ at $270 \mathrm{MeV}$. The upgraded deuteron beam polarimeter has been used to obtain the vector and tensor polarizations during 2016/2017 runs for the DSS experimental program. The polarimeter has been also used to tune the polarized ion source parameters for 6 different spin modes. The data analysis has been performed using the developed software based on $\mathrm{C}++$ language and ROOT5 library.
\end{abstract}

\section{Introduction}

The study of the spin structure of two-nucleon and three-nucleon short-range correlations by means of measuring the polarization observables in the deuteron induced reactions is the main goal of the DSS project at the Nuclotron [1-3]. The high precision polarimetry of the deuteron and proton beams is important for these investigations.

The goal of the present article is to report new results on measuring the vector and tensor beam polarizations using the upgraded polarimeter based on the asymmetry measurements in dp-elastic scattering at $270 \mathrm{MeV}$ [4] at the Internal Target Station (ITS) at the Nuclotron. These measurements were performed during the DSS experiment to study the vector $A_{\mathrm{y}}$, tensor $A_{\mathrm{yy}}$ and $A_{\mathrm{xx}}$ analyzing powers in dp-elastic scattering at large transverse momenta [5].

\section{Deuteron polarimeter at ITS}

Efficient polarimetry can be achieved even at relatively low beam intensity using a thin solid target inside the inner ring of the accelerator. The luminosity can be increased significantly due to multiple beam passage through the interaction point and using of a correctly configured

\footnotetext{
*e-mail: skhomenko@jinr.ru
} 
internal target trajectory. Therefore, the internal beam polarimeter with a very thin target may have approximately the same efficiency as the extracted beam polarimeters.

The polarimeter based on dp-elastic scattering at large angles $\left(\theta_{c m} \geq 60^{\circ}\right)$ at $270 \mathrm{MeV}$ [4], where precise data on analyzing powers [6-8] exist, has been developed on the Internal Target Station at the Nuclotron [9]. The accuracy of determination the deuteron beam polarization achieved by means of this method is better than $2 \%$ because of the values of the analyzing powers obtained for the polarized deuteron beam, where absolute polarization had been calibrated via the $\left.{ }^{12} C(d, \alpha)\right)^{10} B^{*}\left[2^{+}\right]$reaction [8].

Deuteron beam polarimeter [4] is placed in the Nuclotron ring. It consists of a spherical scattering chamber and target change system that can set six different targets. A detector support with 39 mounted plastic scintillation counters is placed downstream the ITS spherical chamber. Each plastic scintillation counter was coupled to a photo-multiplier tube Hamamatsu H7416MOD. Eight proton detectors were installed for the left arm, similar - for the right and upper ones, but due to the space limitation - only four for the lower arm.

The angular span of one proton detector was $2^{\circ}$ in the laboratory system, which corresponds to about $4^{\circ}$ in the cms. Three deuteron detectors are placed at scattering angles of deuterons coinciding kinematically with the protons. Only one deuteron detector can cover the solid angle corresponding to four proton detectors placed down. In addition, one pair of detectors is placed to register two protons from quasi-elastic pp-scattering at $\theta_{\mathrm{pp}}=90^{\circ}$ in the $\mathrm{cms}$ in the horizontal plane. The scattered deuterons and recoil protons at $270 \mathrm{MeV}$ were detected in kinematic coincidence over the cms angular range of $65-135^{\circ}$ at eight different angles, defined by the positions of the proton detectors.

The VME (Versa Module Eurocard) based data acquisition system with new software [10] was used for data taking from scintillation detectors. The signals from the detectors were fed in 16-channel TQDC-16 charge-time-digital [11] converters via the commutator bar. TQDC16 module allows one to measure the amplitude and time of the signal appearance simultaneously. The hardware of the DSS VME system consists of 4 TQDC-16 modules, trigger modules TTCM or TMWR and U40 [11] and VME controller [11]. There is an opportunity to tune the first-level trigger using firmware logic of the trigger and TQDC-16 modules.

A new multichannel high-voltage power supply system based on Wiener MPod have been developed to provide the power for about 70 scintillation detectors equipped with Hamamatsu photomultipliers.

\section{Experiment at ITS}

The ITS is appropriate a study the energy dependence of polarization observables for the deuteron-proton elastic scattering and deuteron breakup reaction with the detection of two protons at large scattering angles. For these purposes the $\mathrm{CH}_{2}$-target of $10 \mu \mathrm{m}$ thick is used for measurements. The yield from carbon content of the $\mathrm{CH}_{2}$-target is estimated in separate measurements using several twisted $8 \mu \mathrm{m}$ carbon wires. The measurements were performed using the ITS at the Nuclotron [9] with a new control and data acquisition system [12].

A new source of polarized ions (SPI) [13] has been used to provide the polarized deuteron beam. In the current experiment the spin modes with the maximal ideal values of $\left(P_{z}, P_{\mathrm{zz}}\right)=$ $(0,0),(-1 / 3,-1)$ and $(-1 / 3,+1)$ have been used. The deuteron beam polarization has been measured at $270 \mathrm{MeV}$.

The DSS data taking was divided in 3 parts: November 2016, December 2016 and February 2017 runs. The deuteron beam polarization measurements were performed at $270 \mathrm{MeV}$ before and after each energy studied in the range of 400-1800 MeV [5]. The polarimeter [4] has been also used to tune other spin modes of SPI. 
ITS

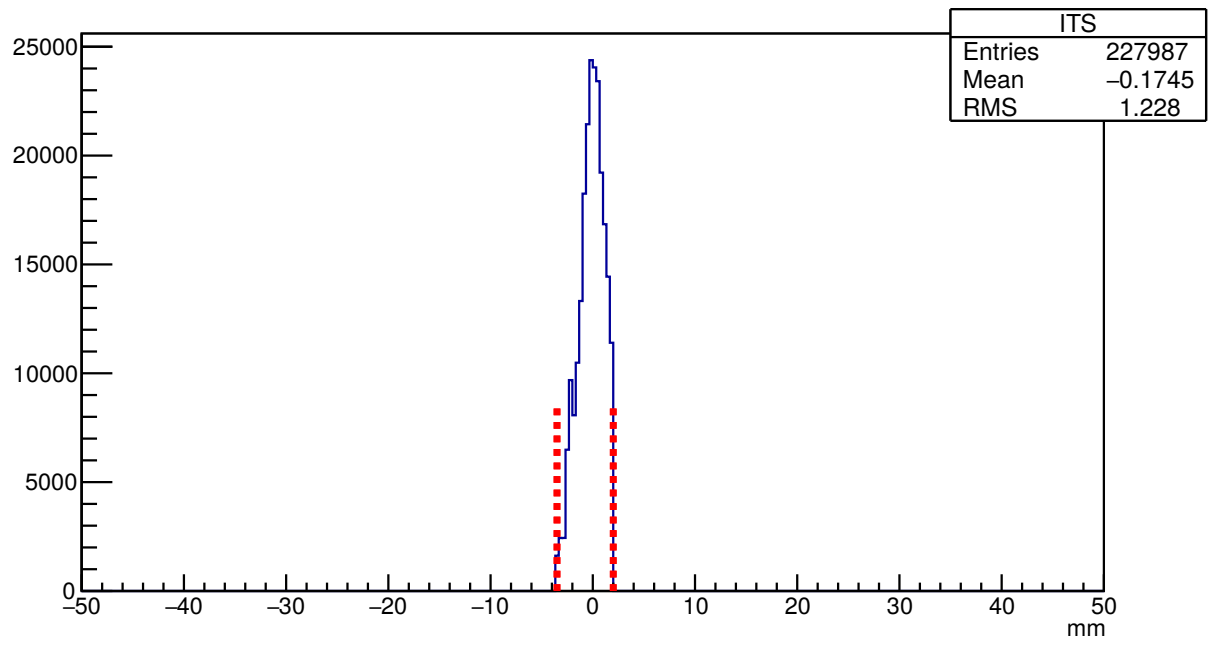

Figure 1. The internal target position selection window
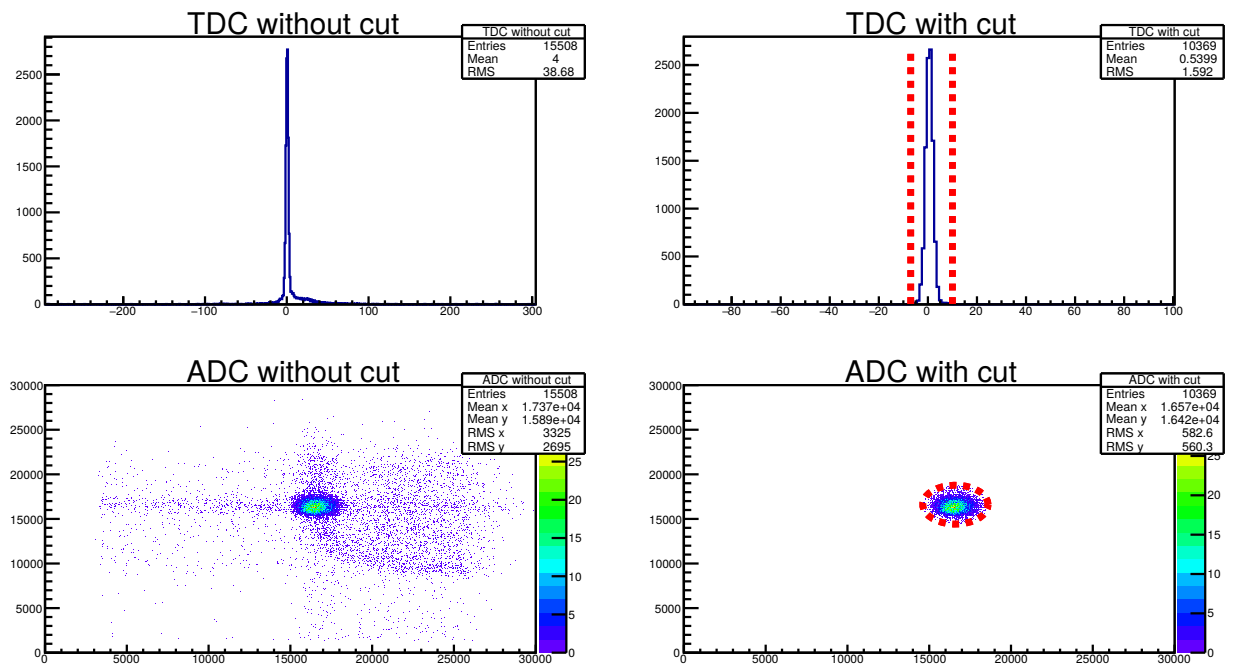

Figure 2. The selection of dp-elastic scattering events using time-of-flight difference and energy losses correlation

The software for the data analysis was carried out in $\mathrm{C}++$ developed using the ROOT package. It consists of several classes and methods. This software can read root representation of the experiment data graphical windows, save vector and raster results, graphics and cuts.

The dp-elastic scattering events at $270 \mathrm{MeV}$ were selected using correlation of the energy losses and time-of-flight difference for the deuteron and proton detectors. The selection of dp-elastic scattering has been performed using graphical windows. 


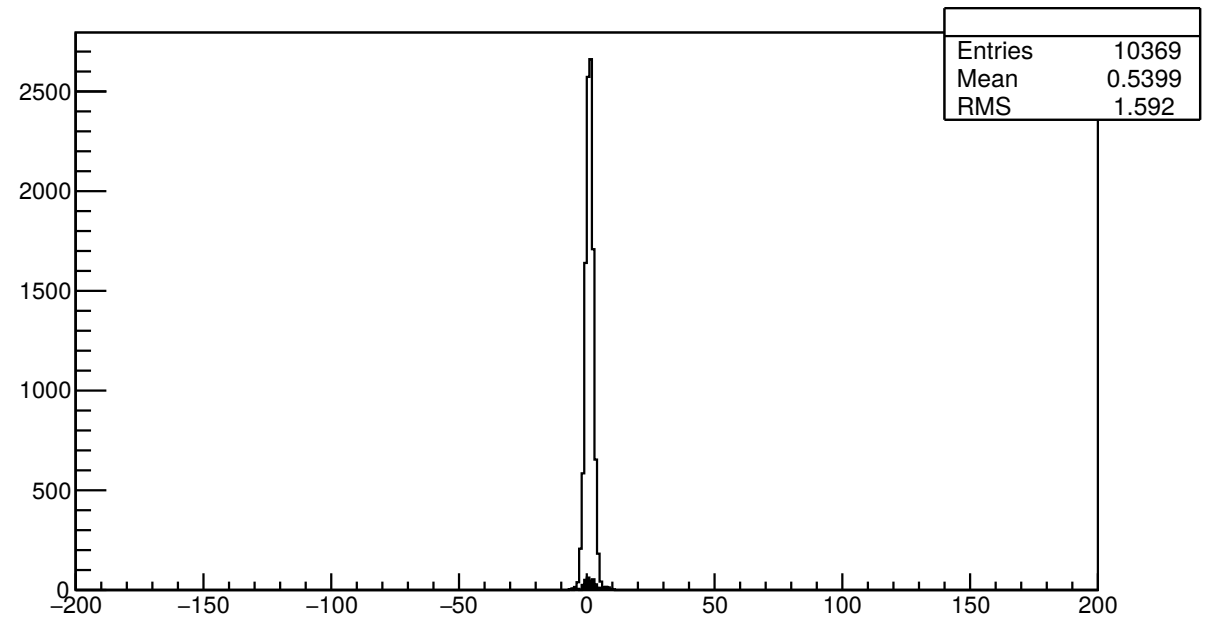

Figure 3. dp-elastic scattering events obtained on the polyethylene and carbon targets

Firstly, the data have been selected by cut for the internal target position (Fig. 1). This criterion rejects all detector signals which come when the target is outside the beam. The selection procedure requires using of only the one graphical window for all pairs of detectors.

The next step is using the graphical windows for time-of-flight difference (Fig. 2). One dimensional histograms obtained for the polyethylene target has a sharp hydrogen peak and wide carbon background.

The final step is the cut to correlate the energy losses (Fig. 2), where we can see 2D histograms with clean hydrogen signal.

The measurements were performed using the $\mathrm{CH}_{2}$ target only. The carbon contamination was measured to be less than about $0.5 \%$ after using all graphical windows (Fig. 3). Therefore, the subtraction of the carbon content should not be applied.

The precise data on the deuteron analyzing powers at $270 \mathrm{MeV}$ ![6-8] have been used to get the polarization values at several angles [4]. Assuming that the Y-axis is a symmetry axis $\left(\beta=90^{\circ}, \varphi=0^{\circ}\right)$ one can calculate $P_{z}$ and $P_{\mathrm{zz}}$ using the normalized dp-elastic scattering events and analyzing powers known [4]. The values of the beam polarization for different spin modes have been obtained as weighted averages for 8 scattering angles for dp-elastic scattering in the horizontal plane only. The typical values of the beam polarization were about $65-75 \%$ from the ideal values.

\section{The results of the polarization measurements}

The vector and tensor polarizations were measured seven, six and four times in the parts in November-2016, December-2016 and February-2017 runs, respectively. The values have small statistical and systematics errors. They are rather stable within each part of the experiment. The exception is the December-2016 part, when the physics program was divided in two parts by tuning the SPI for pure tensor modes $\left(P_{z}, P_{\mathrm{zz}}\right)=(0,-2)$ and $(0,+1)$ during 8 hours. This is the reason why December-2016 part is divided by sets of measurements. The polarization values were approximated by the constants for all four sets of the data. The 

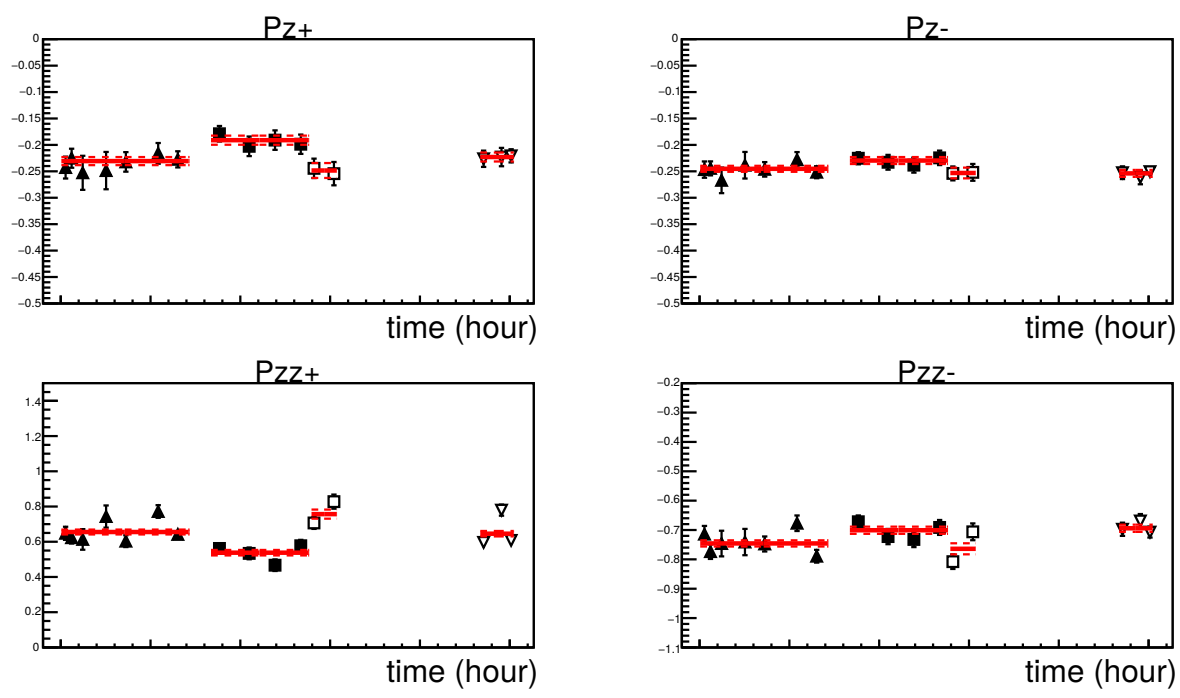

Figure 4. Polarizations values during the runs in 2016 and 2017 yy

Table 1. The vector and tensor polarizations for different spin modes

\begin{tabular}{lllll}
\hline Polarization values $\left(p_{z}, p_{\mathrm{zz}}\right)$ & $P_{z}$ & $d P_{z}$ & $P_{\mathrm{zz}}$ & $d P_{\mathrm{zz}}$ \\
\hline$(-1 / 3,+1)$ & -0.272 & 0.019 & 0.733 & 0.035 \\
\hline$(-1 / 3,-1)$ & -0.272 & 0.014 & -0.793 & 0.026 \\
\hline$(0,+1)$ & 0.040 & 0.023 & 0.725 & 0.042 \\
\hline$(0,-2)$ & 0.042 & 0.013 & -1.478 & 0.030 \\
\hline$(-1,+1)$ & -0.648 & 0.016 & 0.628 & 0.027 \\
\hline$(-2 / 3,0)$ & 0.468 & 0.013 & 0.039 & 0.021 \\
\hline
\end{tabular}

results of the measurements and approximation are presented in Fig. 4. All the results are within two standard deviations from these constants. One can see that the beam polarization values are quite stable within more than 200 hours of the SPI operation. On the other hand, SPI has demonstrated a good reproducibility of the polarization values for different sets of the data after long interruptions. The typical values of the vector and tensor components of the beam polarization for the spin modes $(-1 / 3,-1)$ and $(-1 / 3,+1)$ are given in Tab. 1 .

The polarimeter [4] has been also used to tune the SPI operation for pure tensor spin modes $(0,-2)$ and $(0,+1)$, for pure vector spin mode $(-2 / 3,0)$ and for the spin mode $(-1,+1)$ with both vector and tensor components. Preliminary results are presented in Tab. 1 . One can see, that the typical values of the beam polarization were about $65-75 \%$ from the ideal values for all 6 spin modes of SPI.

\section{Conclusion}

The upgraded version of the $270 \mathrm{MeV}$ deuteron beam polarimeter was used to obtain the vector and tensor polarizations during 2016/2017 runs. The long-term stability of the vector 
and tensor components of the beam polarization has been demonstrated for the spin modes $(-1 / 3,+1)$ and $(-1 / 3,-1)$ of SPI. The polarimeter has been used for tuning the polarized ion source parameters for 6 different spin modes.

Acknowledgments. The authors thank the Nuclotron staff for providing good conditions of the experiment. They thank V.B. Shutov for the help with the SPI tuning. They express the gratitude to V.I. Maximenkova for the help during the preparation of the detector and S.N. Bazylev, I.V. Slepnev, V.M. Slepnev and A.V. Shutov for the design of the trigger logic firmware for VME DAQ system. The work has been supported in part by the RFBR under grant $N^{0} 16-02-00203 a$, by the Ministry of Education, Science, Research, and Sport of the Slovak Republic (VEGA Grant No. 1/0113/18), by JINR- Slovak Republic and JINRRomania scientific cooperation programs in 2016-2018.

\section{References}

[1] V. P. Ladygin et al., Phys.Part.Nucl. 327, 45 (2014)

[2] V. P. Ladygin et al., Few Body Syst. 709, 55 (2014)

[3] M. Janek et al., Few Body Syst. 58, 40 (2017)

[4] P. K. Kurilkin et al., Nucl.Instr.Meth. in Phys.Res. A 642, 45 (2011)

[5] V. P. Ladygin et al., talk at this Conference

[6] K. Sekiguchi et al., Phys.Rev. C 65, 034003 (2002)

[7] K. Sekiguchi et al., Phys.Rev. C 70, 014001 (2004)

[8] K. Suda et al., Nucl.Instr.Meth. in Phys.Res. A 745, 572 (2007)

[9] A. I. Malakhov et al., Nucl.Instrum.Meth. in Phys.Res. A 440, 320 (2000)

[10] A. Yu. Isupov, JPCS 938, 012019 (2017)

[11] http://afi.jinr.ru

[12] A. Yu. Isupov et al., NIMA 689, 127 (2013)

[13] V. V. Fimushkin et al., J.Phys.Conf.Ser. 678(1), 012058 (2016) 